\title{
The 2020 Research on Problematic Information on the COVID19 Pandemic. A Systematic Literature REVIEW
}

\author{
Nicola Righetti \\ Department of Communication \\ University of Vienna
}

\author{
Luca Rossi \\ Digital Design Department \\ IT University of Copenhagen \\ lucr@itu.dk
}

\author{
Giada Marino \\ Department of Economics and Business \\ University of Sassari
}

gmarino@uniss.it

July 8, 2021

\begin{abstract}
The COVID-19 pandemic has been a major turning point in scholarly attention to informationrelated problems, including the infodemic and fake news. The paper presents a systematic and comprehensive literature review on multidisciplinary research into problematic information around COVID-19 published in 2020, with a view to identifying the main trends from a disciplinary, methodological, and substantive perspective. We collected 862 records in English from three leading scientific databases (Scopus, Web of Science, and EBSCOhost) by searching, in the title and abstract, a set of keywords related to COVID-19 and information problems. After removing the duplicates and documents other than scientific papers published in scientific journals (such as magazine articles and letters), the three authors screened the records to retain the empirical articles which dealt more than just incidentally with the topic, ending up with 378 papers. The three coders analyzed the results and applied a number of pre-defined categories related to the disciplinary, methodological, and substantive characteristics of the papers. Analysis of frequencies and computational methods, including social network analysis and text mining, were used to analyze the data. The corpus of 378 papers published in 2020 on problematic COVID-19 information revealed considerable contributions from Medicine and Social Sciences and a disciplinarily and geographically interconnected field. Quantitative methods and especially surveys stand out as the most popular approaches, with a considerable number of more discursive papers offering expert views on pandemic-related informational problems. The main trends from a substantive perspective were conspiracy theories and their impact on norm compliance, and the attention to informational problems defined though the concept of infodemic.
\end{abstract}

Keywords Disinformation · Misinformation · Indodemic · COVID19 · Pandemic

\section{Introduction}

A major turning point in scholarly attention to fake news after the 2016 U.S. Presidential elections has certainly been the COVID-19 pandemic. Threatening both the health of the public and of democracy itself, 
unreliable and misleading information earned a place among the central issues in communication science, besides attracting increasing attention from other disciplines - first and foremost health-related sciences with its worrisome consequences.

The spread of a worldwide contagion caused by a previously unknown virus has radically changed everyday lives and exposed people to unprecedented levels of uncertainty, driving them to seek information to soothe their anxieties. A torrent of news has met this need (Bermejo, 2020; Zarocostas, 2020), alongside institutional communication (Lovari, 2020), and also unsubstantiated, ideologically and economically motivated mis/disinformation (Brennen et al., 2020; Ferrara, Cresci, \& Luceri, 2020; Giglietto et al., 2021), state-backed information operations (DiResta et al., 2020; Molter \& DiResta, 2020; Serrato \& Schafer, 2020), and conspiracy theories (Shahsavari et al., 2020).

When individual behaviors are the clue to stemming the spread of a problem and avoiding a possible catastrophe, reaching people with the right information is among the most strategical assets. Therefore, institutions and scholars were understandably alarmed by a poisoned information flow that threatened to hinder the implementation of measures aimed at flattening the infection curve. This led to frenetic research activity, resulting in an exceptional quantity of specialist studies. The importance of this corpus of scholarly research, and the fact that such an amount of information can only be handled with difficulty during a normal research activity, convinced us of the need to start a systematic mapping and analysis of this field to favor its further development.

A review of prior literature is an essential feature of any research, creating a foundation for advancing knowledge by facilitating theory development, closing over-researched areas and uncovering under-explored areas (Webster and Watson, 2002). It also allows scholars to reflect critically on the academic construction of the discourse (Neumayer and Rossi, 2016), which is especially needed when a topic trends up fast. Substantive insights are not alone in being of interest for the development of a discipline; methodological and disciplinary insights can be equally valuable. The interdisciplinarity character of certain topics (and problematic information is certainly one of these) requires the efforts of a multidisciplinary network of scholars capable of pooling their different perspectives to obtain good results. In exceptional circumstances such as the COVID-19 pandemic, moreover, new constraints on research emerge, like the methodological limitations imposed by social distancing measures.

It can therefore be appropriate to analyze this corpus of research by taking a step back. As a first move in this direction, we present an analysis of a comprehensive set of papers $(\mathrm{N}=378)$ published in 2020 on COVID-19 and problematic information, collected through a systematic review of the literature, with the aim of providing insights into the disciplinary structure, methodological approaches, and substantive focuses of the field.

\section{Problematic information amidst a pandemic}

Digital communication technologies had already reached a turning point in the production, circulation and social impact of rumors, misleading and false information when their pernicious influence was further exacerbated by the outbreak of the pandemic (Lovari \& Righetti, 2021). Digital media changed the information market by lowering barriers and controls regulating its entrance and creating significant earning potential for information producers. The result was to foster an increasingly competitive environment which has given rise to the exploitation of emotionally overcharged, exaggerated, controversial, polarizing and even fabricated stories to increase profit (Stanyer, 2009; Sunstein, 2018). At the same time, publics have become producers and the legitimacy crisis affecting expert knowledge, also with particular reference to health-related knowledge (Kata, 2012), has favored the spread of unscientific beliefs. Scholars, journalists and communication practitioners have referred to this kaleidoscopic information environment by using a variety of terms (Jack, 2017), such as fake news, misinformation, disinformation, information disorder, but also conspiracy theories, and, more recently, infodemic. In this section we introduce and provide concise definitions of the main terms for the study of problematic information which have been the starting point for our research.

\subsection{Fake News, Misinformation and Disinformation}

Fake news is one of the most popular ways of referring to mis/disinformation (Righetti, 2021). The term has been defined as "fabricated information that mimics news media content in form but not in organizational process or intent" (Lazer et al., 2018, p. 1094). In the last few years, scholars have exposed the multi-faceted aspects of the phenomenon, critically analyzing their characteristics (Giglietto et al., 2019; Venturini, 2019). 
The term "fake news" gained popularity along with "post-truth". Declared the Word of the Year in 2016 by the Oxford Dictionary, it refers to the phenomenon according to which "objective facts are less influential in shaping public opinion than appeals to emotion and personal belief" (Flood, 2016). Post-truth politics refers to the conduct of political actors to whom the internet and social media have provided new means of communication and who adapt their narrative to the sentiment of the public which is constantly monitored through an incessant flow of "big data". By tracking and orienting people according to emotion, instead of reason, fake news and post-truth make it possible "to live in a world of data but no facts" (Davies, 2016).

Despite being used in a very general sense to cover the whole phenomenon of false information, fake news is just one among many types of problematic information which scholars have focused on. In particular, experts have distinguished between different types of problematic information based on the different intentions behind its spread and creation. Among the most common distinctions is the one between misinformation, indicating false news spread unintentionally (for instance by journalists who fail to verify their sources or by lay people due to ignorance), and disinformation, referring to the deliberate fabrication and spread of false news (Jack, 2017). Despite the conceptual simplicity of the distinction, it has been observed that the boundaries between the two types tend to blur in the concrete online communication system (Giglietto et al., 2016), and also that true information can be used to harm. Disinformation can be further distinguished based on its ideological (e.g.: political propaganda), or economic purpose (e.g.: a newspaper publishing fake news to make a profit by attracting visitors to its website). This too is a distinction that blurs in the real information environment where highly partisan fake news is a potential source of earnings independently from any authentic ideological interest (Subramanian, 2017). Recently, the more general concept of pseudo-information has been suggested to include all types of false or inaccurate information which can result in harmful social consequences (Kim \& de Zúñiga, 2020).

\subsection{Infodemic and Information Disorder}

Although problems related to information are as old as humanity, social media in general, and specific social and historical circumstances in particular, have captured the attention of the public, favoring the emergence of neologisms. Post-truth is an example of these new terms which have emerged in relation to politics. Another example, regarding health communication, is infodemic. This term has been used by the World Health Organization to refer to the excessively prolific, confused and potentially harmful information flow on COVID-19. The term is not totally new, since it was used for the first time in a Washington Post comment about SARS by David J. Rothkopf, meaning: "A few facts, mixed with fear, speculation and rumor, amplified and relayed swiftly worldwide by modern information technologies" which "have affected national and international economies, politics and even security in ways that are utterly disproportionate with the root realities" (Rothkopf, 2003).

The WHO defined infodemic as "too much information including false or misleading information in digital and physical environments during a disease outbreak. It causes confusion and risk-taking behaviors that can harm health. It also leads to mistrust in health authorities and undermines the public health response. An infodemic can intensify or lengthen outbreaks when people are unsure about what they need to do to protect their health and the health of people around them. With growing digitization - an expansion of social media and internet use - information can spread more rapidly. This can help to more quickly fill information voids but can also amplify harmful messages"[^https://www.who.int/health-topics/infodemic\#tab=tab_1.]. By immediately bringing to mind the word epidemic, infodemic is a term framing online information as a public health concern: digital messages going "viral" spread false information among people, threatening the health of society. As a consequence, and in analogy to evidence-based medicine, "Infodemic management is the systematic use of risk- and evidence-based analysis and approaches to manage the infodemic and reduce its impact on health behaviors during health emergencies"[^Ibidem.].

Since the circulation of content on the Internet and social media is controlled by algorithms, social media platforms play a significant role in countering the infodemic. These platforms have implemented a number of initiatives aimed at curbing the uncontrolled flow of misinformation and disinformation on COVID-19, such as monitoring the use of related keywords, taking down fake news which poses risks to the population, pointing users to official sources from which to obtain reliable information, and working more closely than ever before with government and public health institutions (Lovari, Martino, Righetti, 2019).

\subsection{Conspiracy Theories}

The uncertainty brought about by the pandemic has been a breeding ground for conspiracy theories (Grey Ellis 2020). If all the types of misinformation and disinformation are dangerous for society and individuals, 
conspiracy theories are especially harmful, since they can negatively impact health behaviors, diminish well-being, erode trust in institutions and threaten social cohesion (Douglas et al. 2019). Scholars found that conspiracy theories are linked to feelings of powerlessness, uncertainty, existential anxiety, lack of control over the situation, and can therefore arise in times of social, political and psychological crisis such as the COVID-19 pandemic: "When a terrible event has occurred, acceptance of such theories may justify or rationalize the affective state produced by that event" (Sunstein \& Vermeule, 2009).

Conspiracy theories fulfill psychological and sociological needs such as the need for security, control and meaning, but far from being healthy coping strategies, they lead to undesirable consequences from a social and individual point of view (Douglas et al. 2019) These comprise behaviors prejudicial to health (Douglas et al. 2019) including specific COVID-19-related behaviors such as low compliance with social distancing measures (Swami and Barron 2020), racist attitudes towards social groups and minorities (Grey Ellis 2020; Schild et al. 2020; Shimizu 2020), diminished trust in science and in the institutions as a result of fostering anti- and pseudo-scientific attitudes (Douglas et al. 2019), the potential erosion of democracy by thus reducing political participation (Butler, Koopman, and Zimbardo 1995; Uscinski and Parent 2014), and disruptive and violent behavior in general (Hamilton 2020; Ahmed et al. 2020). Research Questions The main aim of this research was to identify and describe the multifaceted literature on problematic information in the context of the COVID-19 pandemic from three main perspectives, asking:

- RQ1) What is the scientific structure of this field of study?

- RQ1a) In which scientific disciplines was the research conducted?

- RQ1b) What was the level of interdisciplinarity?

- RQ1c) What was the degree of geographical interconnection?

- RQ2) What methodological approaches have been used?

- RQ2a) What methodological approaches and analysis techniques were employed?

- RQ2b) How representative were the samples used to answer the research questions, and how much attention was devoted to the quality of the analysis?

- RQ2c) What social media platforms and online services were used to conduct the research?

- RQ3) What are the main topics covered?

- RQ3a) What were the main terms used to refer to problematic information?

- RQ3b) What were the main research topics?

- RQ3c) Which countries were taken into consideration in the analyses?

\section{Materials and Methods}

To collect and screen the literature we followed the PRISMA guidelines for systematic reviews and metaanalyses (Page et al., 2021), already profitably used to study health misinformation on social media (Wang, McKee, Torbica, \& Stuckler, 2019). The PRISMA guidelines consist of methodological steps and of a set of items for conducting and reporting systematic literature research. The information flow characterizing the different phases of a PRISMA systematic review includes three main steps, namely identification, screening, and eligibility, resulting in the final records which will be investigated. These steps are described below and summarized in Figure 1.

\subsection{PRISMA Workflow}

\subsubsection{Identification}

Four leading academic databases Scopus, Web of Science, EBSCOhost Communication \& Mass Media Complete, and EBSCOhost Communication Abstracts, were queried using the following keywords:

i. COVID OR COVID-19 OR coronavirus

ii. misinformation OR disinformation OR malinformation OR "problematic information" OR "fake news" OR "information disorder" OR infodemic OR conspiracy OR rumor OR hoax. 

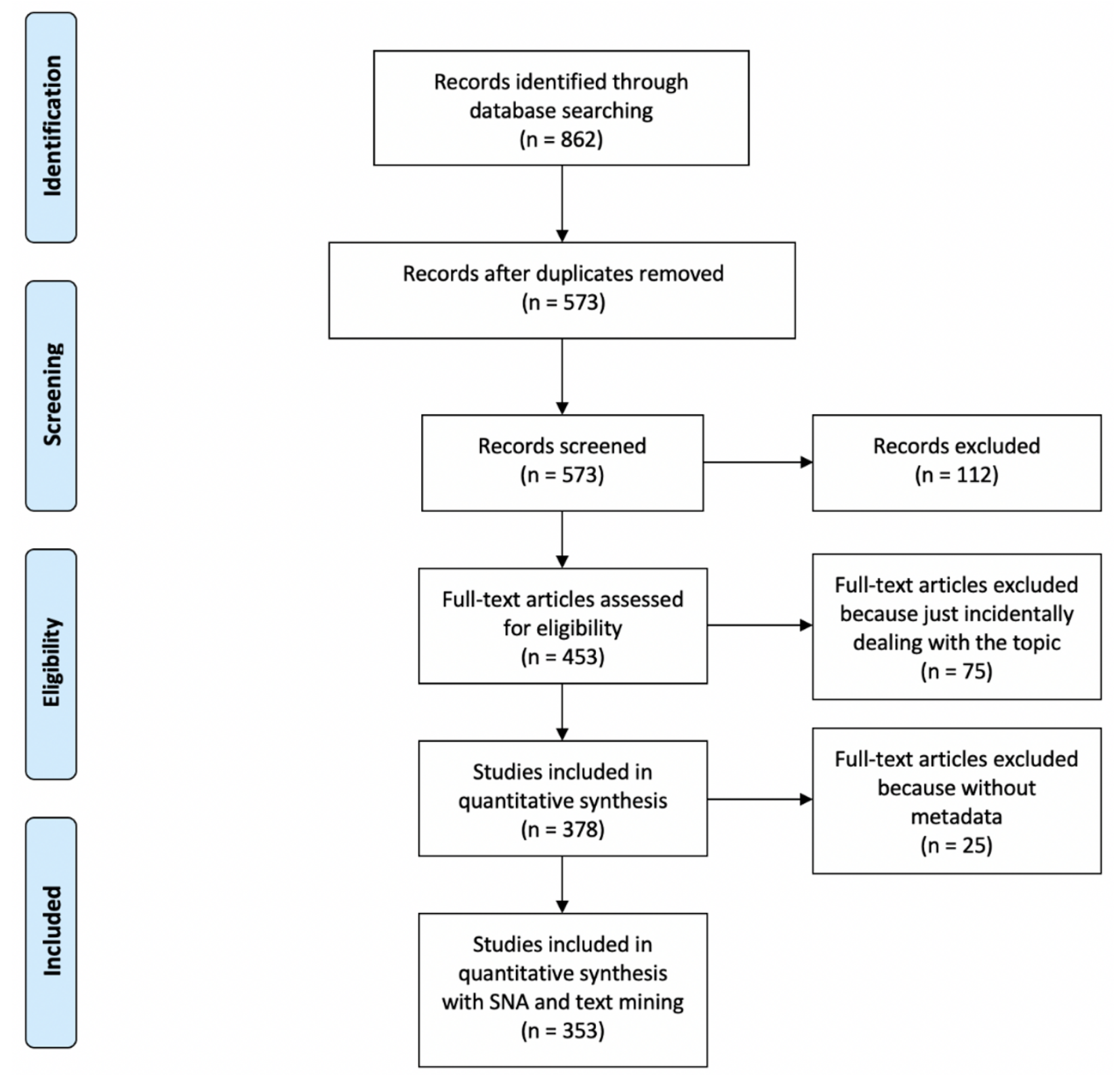

Figure 1: PRISMA flow chart of the different phases of the systematic review.

The terms were searched in the title and abstract of articles (excluding commentaries, letters, reviews etc., since the focus was mainly on original empirical research), published in 2020 and written in English, when the COVID-19 pandemic started. This research yielded 482 records from Scopus, 351 from Web of Science Core Collection, and 29 from both EBSCOhost Communication \& Mass Media Complete and EBSCOhost Communication Abstracts. Duplicates were identified and removed, resulting in a total of 573 . We evaluated the completeness of this data set against the number of records in the literature research service of the World Health Organization, "a comprehensive multilingual source of current literature" on COVID-19, which returned, by using the same search string, 484 results[^https://www.who.int/emergencies/diseases/novelcoronavirus-2019/global-research-on-novel-coronavirus-2019-ncov/].

\subsubsection{Screening and Assessment of Eligibility}

The records were subsequently screened by the authors. In order to keep the focus on original research, documents like introductions to special issues, articles in magazines, and letters, and papers not mentioning 
the keywords were automatically removed by relying on metadata and screening the records without abstracts (which were often found to be magazine articles or letters), resulting in 453 records. These records were further analyzed by the three authors, and, after a close inspection, those considered not relevant because dealing only incidentally with the key topics of problematic information, were removed. The intercoder reliability was measured with Krippendorff's alpha (Krippendorff, 2004) on a random sample of 50 records (representing over $10 \%$ of the 453 records) resulting in a value of 0.885 , which is considered a high level of reliability. At the end of the process 378 records remained. All the records were coded and included in a first quantitative synthesis. The network analysis and text mining analysis, which made use of metadata available from Scopus and Web of Science, was based on the 353 papers including the necessary information.

\subsection{Methods}

Recognizing the interdisciplinary and global nature of the problem at issue, we assessed the structure of the field based on the disciplinary and geographic boundaries and interconnections. The disciplinary areas were identified by matching the journals with data from SCImago Journal \& Country Rank, "a publicly available portal that includes the journals and country scientific indicators developed from the information contained in the Scopus database [whose] indicators can be used to assess and analyze scientific domains" . To answer the other research questions, we relied on social network analysis and the R package "bibliometrix" (Aria \& Cuccurullo 2017). In particular, the interdisciplinary connections were analyzed through bibliographic coupling (Kessler, 1963). The network of scientific collaborations, and their relations with geographical areas, were also investigated. These analyses were based on metadata provided by Scopus and were therefore made on the 353 papers (93.4\% of the entire dataset) available on this database.

With regard to the substantive content, we started by analyzing the most common terms for problematic information, based on the consideration that the terms used to define phenomena are not neutral, but often convey a particular perspective on problems. We also analyzed the topics and their prevalence across the corpus of research, exploring possible differences in relation to disciplines, through text mining techniques. Text mining is a useful approach to summarize and extract information from a large quantity of documents. Although it can hardly attain to the same level of insight as a human being thoughtfully reading the texts, it can identify the main trends characterizing a large corpus of texts. The textual data were pre-processed consistently with similar research on large corpora of scientific literature (e.g.: Chan, Grill, 2020), focusing on the abstract of papers, which is particularly useful for this purpose since the abstract's function is precisely to summarize the main content of a paper. Text mining results can be more or less clear or blurred, depending on the complexity of the corpus, thus to get more robust results we relied on two different methods: descending hierarchical classification, a method developed by Reinert (Reinert, 1983, 1990) and implemented computationally in the free software Iramuteq by Ratinaud (2008) that extracts lexical clusters representing distinct "semantic worlds", or relatively homogeneous semantic areas, and structural topic modeling (Roberts, Stewart, Tingley, 2019), a method that allows researchers to discover topics, their prevalence in the corpus, and estimate possible relationships with covariates.

While the first method automatically extracts the optimal number of clusters, the second requires the researcher to specify the desired number of topics. This choice has a high margin of subjectivity and its validity is dependent on the specific structure of the dataset. Generally speaking, the higher the number of topics, the higher their "granularity". We fitted several models, systematically varying the number of topics, ascertained the robustness of the results (the same topics, at different levels of granularity, tended to emerge) and found that 15 topics was a sufficient number to capture the main trends, ensuring conciseness and avoiding too much redundancy (the "fragmentation" of a single theme in several topics). Besides applying computational techniques using metadata provided by Scopus, a large amount of information was collected by the three authors through an in-depth analysis based on a codebook agreed upon by the coders. As for the screening of the papers, the intercoder reliability was assessed through Krippendorff's alpha (Krippendorff, 2004) on a random sample of 50 records (over $10 \%$ of the dataset). A number of categories were annotated for each paper. The coding procedure was based on the abstract and the full-text, where the necessary information was not contained in the abstract in sufficient detail.

The information collected through this analysis was, among other things, about methodological approaches, distinguishing between quantitative, qualitative, and discursive papers - mainly papers providing informed analysis based on previous literature and available data (Krippendorff's alpha $=0.924$ ); methods, including, for instance, survey, qualitative content analysis, social network analysis, and others (Krippendorff's alpha $=$ 0.82); studied subjects, distinguishing, for instance, between health professionals, general population, social media accounts and others (Krippendorff's alpha $=0.777$ ); countries representing the focus of the study (Krippendorff's alpha $=0.863$ ); social media platforms taken into consideration (Krippendorff's alpha $=$ 


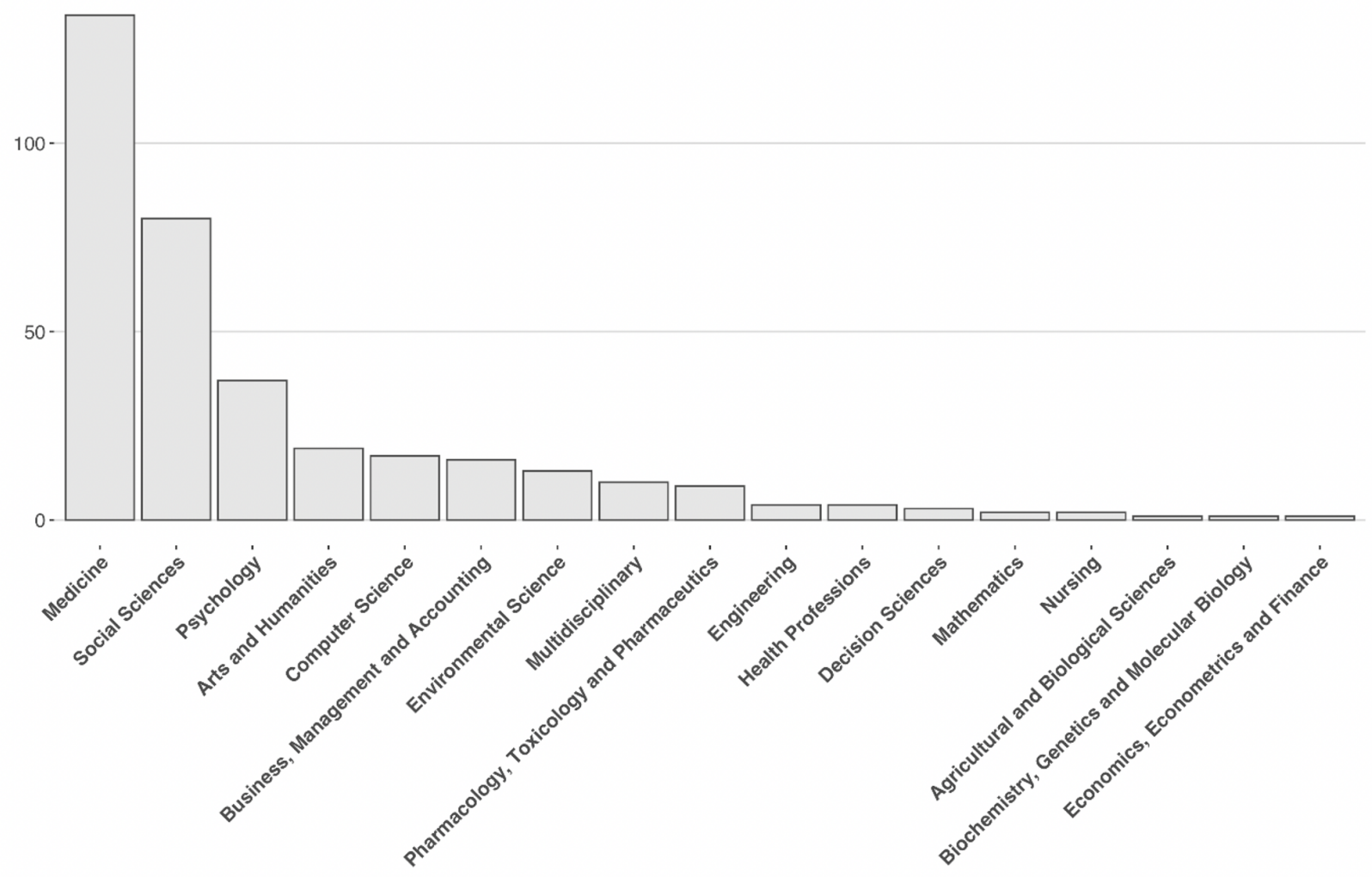

Figure 2: Number of articles published in journals associated with a specific field.

1.00); and online services and social media used to collect data, like Amazon Mechanical Turk (Krippendorff's alpha $=0.794)$. Recognizing the importance of well-grounded methodological research for the development of the field, we also collected information on sampling methods (Krippendorff's alpha $=0.812$ ), sample sizes (Krippendorff's alpha $=0.798)$ and the attention paid to methodological aspects which are important in determining the quality of the results, such as the power analysis (Cohen, 1977) (Krippendorff's alpha $=$ $1.00)$.

\section{Results}

\subsection{Scientific Structure}

Using Scimago we associated each journal with an academic area of research. While these areas are undoubtedly large, they can still be used as a valuable proxy to identify where research dealing with COVID-19 and problematic information was being published. Figure 2 shows the number of articles published in journals associated with a specific field. While there are 17 academic fields represented (including journals listed explicitly as "multidisciplinary"), Medicine and Social Sciences take the lion's share, accounting for 214 papers out of 353 .

A look at the top 5 most represented fields confirms the complex multidisciplinary dimension of the problem that clearly includes medical and social science aspects but also reaches out to psychology, humanities, and computer science. Within this perspective it might come as a surprise that multidisciplinary journals (e.g.: PLOS One) collected only 10 articles.

Given the short life span of the papers that comprise our selection, the use of co-citation to identify disciplinary clusters of papers proves problematic. Co-citation requires papers to be available and to have been read and cited. This seems unrealistic for papers that have been published, at the outside, for less than a year before we ran the analysis/before the date set for the analysis). An alternative strategy to identify disciplinary clusters is bibliographic coupling (Kessler M. 1963). Papers are bibliographically coupled when two works 
reference a common third work in their bibliographies and it is customarily used as a sign that the two works deal with a common subject matter. By performing a bibliographic coupling of the dataset, we obtain a coupled undirected network of 353 and 18,995 edges. The graph is relatively dense (0.30), meaning that it contains several of the possible edges. The graph also includes 34 components, this means that there are 34 groups of papers not connected across the groups. These components have, as expected, different sizes and the largest component ("giant component") accounts for 282 articles, the second largest for 38 and the remaining components are isolated nodes except for couples of two papers.

When it comes to the different scientific fields that are present within the components, there are few differences between the largest and the second largest component, as showed by Figures 3A and 3B. The largest component, that alone includes more than two thirds of the nodes, replicates the picture we observed for the whole network; the second largest component sees a larger presence of Social Science papers and a smaller presence of Medicine papers.

A deeper analysis of the larger component reveals some interesting characteristics: The plot of the k-core decomposition (Figure 3C) shows more than $60 \%$ of the component's nodes $(n=182)$ are part of a clique with a coreness of 181 . Coreness is a measure that helps identify tightly connected groups within a network. A k-core is a maximal group of nodes (papers in our case), all of which are connected to at least other $\mathrm{k}$ nodes. A node in the k-core group must be connected to at least $\mathrm{k}$ other nodes in the group. Within our context, this means that there are 182 nodes sharing one or more bibliographical references and they are from all the academic fields present in our dataset (Figure 3C) showing the existence of a set of shared bibliographical references across the various disciplines.

The 353 papers in our dataset have been written by 932 authors. The affiliations of the authors are predominantly from the United States (199 authors), the UK (68), China (58) and India (56). Figure 4 shows the distribution of the papers in our dataset across the countries.

Level of international collaboration, as well as number of authors, can vary according to disciplinary traditions. To explore this dynamic, we defined an index of international collaboration (IC) within the academic discipline. The index is defined as the sum of the number of distinct countries that participated in the papers within a given discipline, divided by the number of papers in that discipline. The IC score would be 1 if all the papers were written within the same country.

It is interesting to observe that, except for few disciplines - with a small number of articles - that are characterized by an extremely high level of international collaboration, most of the disciplines show some degree of international collaboration/cooperation (Figure 5). If we focus on the three disciplines that are dominant in our dataset (Medicine, Social Sciences and Psychology), Medicine features a higher level of international collaboration than the other two, and Social Sciences score one of the lowest values in the whole dataset (Figure 5). An alternative way to explore international collaboration is to build a country collaboration network where two countries will be connected if two authors affiliated with institutions based in those countries are co-authors of a paper. The network contains 61 nodes (countries) connected by 515 edges. Figure 6 shows the networks with the nodes colored according to their geographic region (following the WHO organizational division into "regional offices"[^https://www.who.int/about/who-we-are/regional-offices]).

While some specific collaborations between various countries are visible (e.g.: the collaboration between the United States and the United Kingdom) there is no visible preference towards collaborating within the geographic regions. We tested this using the value of nominal assortativity (Newman 2002) which measures the tendency of the nodes to connect with other nodes given a specific attribute. The nominal version of assortativity is commonly used to measure the level of homophily existing within network structures. In our case, the result (0.04) suggests a network that is neither assortative nor disassortative for the regional variable and where the regional dimension plays no role.

The analysis of the k-core structure of the country collaboration network shows the presence of a core group of 27 countries (coreness $=16$ ) which are countries marked by a higher degree of international collaboration - as well as smaller groups of countries moving towards the periphery of the network (Figure 7). Regional differences do not seem to play any role in deciding if the country belongs to the core or to the periphery of the network.

\subsection{Methodological Approaches}

Studying pandemic-related problematic information has represented a challenge to researchers due both to the fuzziness and rapid evolution of the phenomenon and to the limitations imposed by social distancing and lockdown measures which have inevitably affected methodological choices. The analysis (Figure 8) 

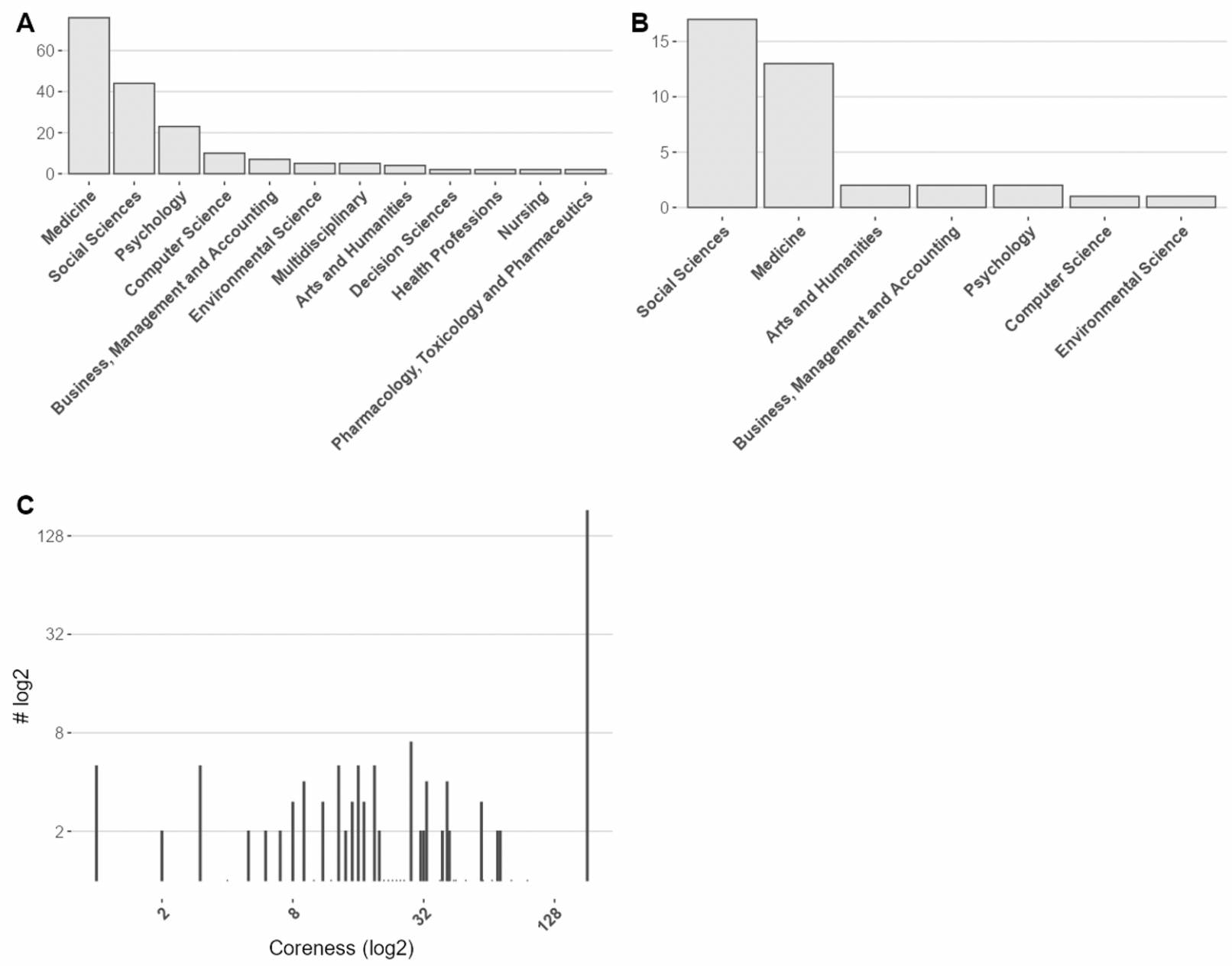

Figure 3: A) Number of papers from different disciplines in the giant component; B) number of papers from different disciplines in the second largest component, C) k-core decomposition of the largest component.

showed that most of the studies were based on a quantitative approach (36\%), a considerable part relied on a discursive approach (30.2\%), and just a minor part, half of those adopting a quantitative approach, were based on a qualitative approach (16.6\%). We also found a residual number of papers presenting datasets shared with the scientific community $(0.7 \%)$.

Focusing on the set of papers based on a qualitative or quantitative approach, it can be noticed that the survey is the most popular approach (120 papers, $49 \%$ of the empirical-based papers), followed by content analysis $(20.8 \%)$, natural language processing $(10.2 \%)$, interviews $(7.3 \%)$, time series analysis $(4.9 \%)$, social network analysis (3.3\%). Agent-based modeling (1.2\%), case studies (1.2\%), ethnographies $(1.2 \%)$, and round tables $(0.8 \%)$ are used in just a few pieces of research.

Sampling choices are important to ensure the validity and generalizability of results. This is especially true with regard to a deductive approach like surveys, generally aimed at testing hypotheses or estimating the prevalence of phenomena, while it is less important for qualitative research which can attain significant results through in-depth analyses of few cases. Focusing therefore on the sample used in studies based on surveys (n $=120$ ), we found that a large majority relied on convenience samples $(77.5 \%)$ - for instance, collected via online services like Amazon Mechanical Turk (8.3\%) or by sharing the link to the survey on social media - followed by quota samples $(19.2 \%)$, and probability samples $(3.3 \%)$. Surveys were more focused on the general population $(75.4 \%)$ than specific social categories, with just a residual percentage based, for instance, on students $(9 \%)$ or health professionals $(5.7 \%)$. 


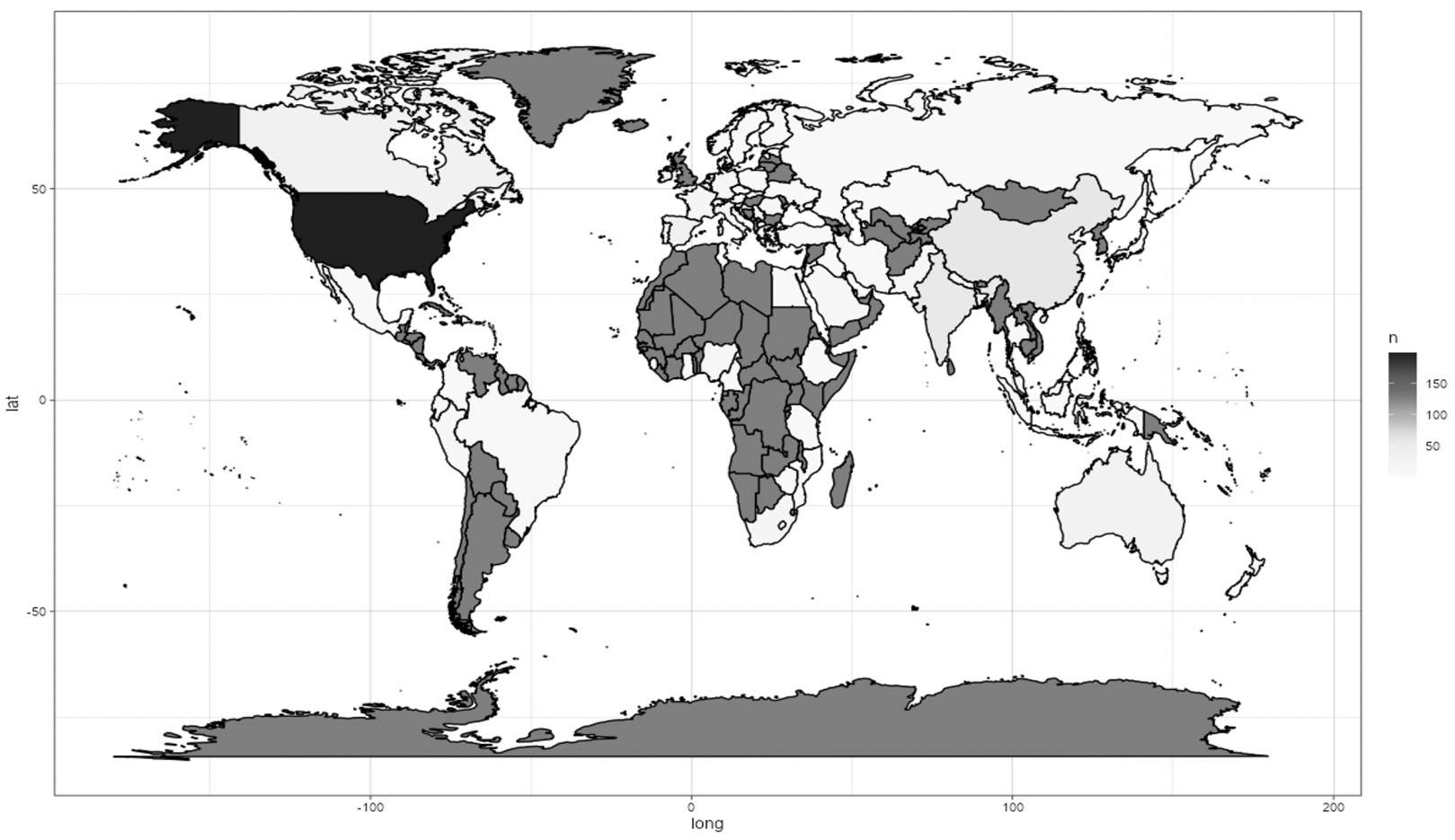

Figure 4: Distribution of papers across countries.

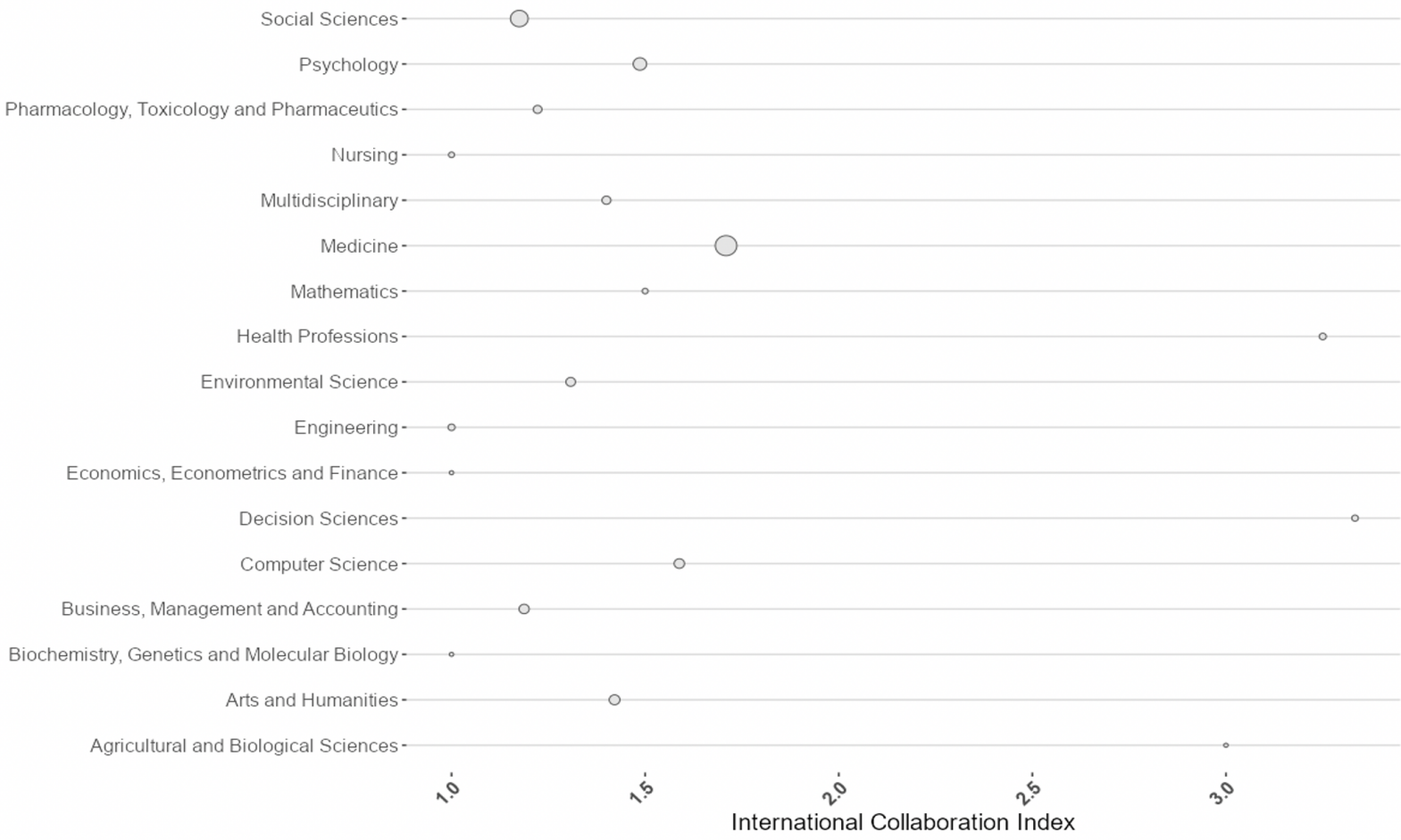

Figure 5: International Collaboration Index per discipline. 


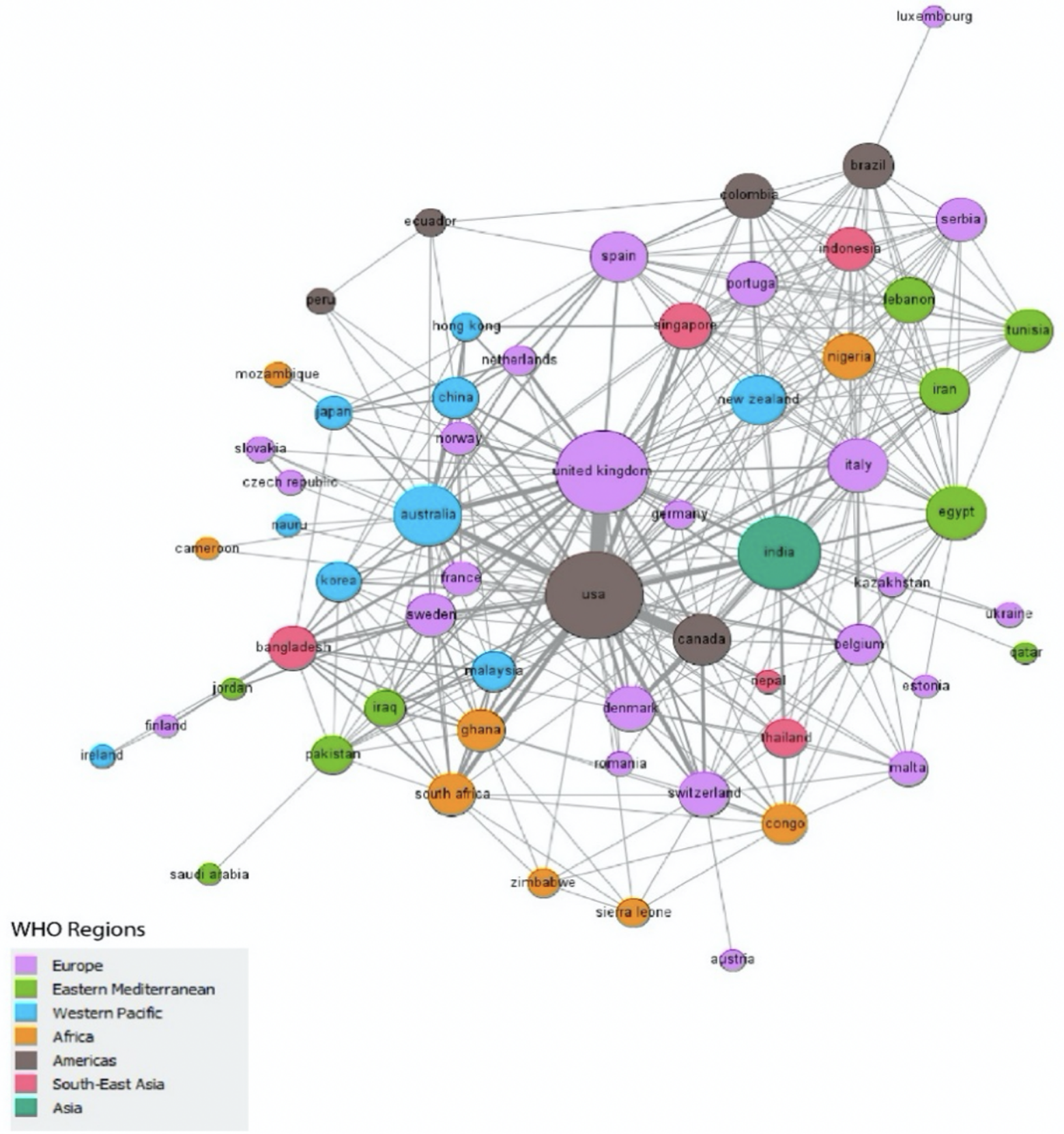

Figure 6: Countries collaboration network. 


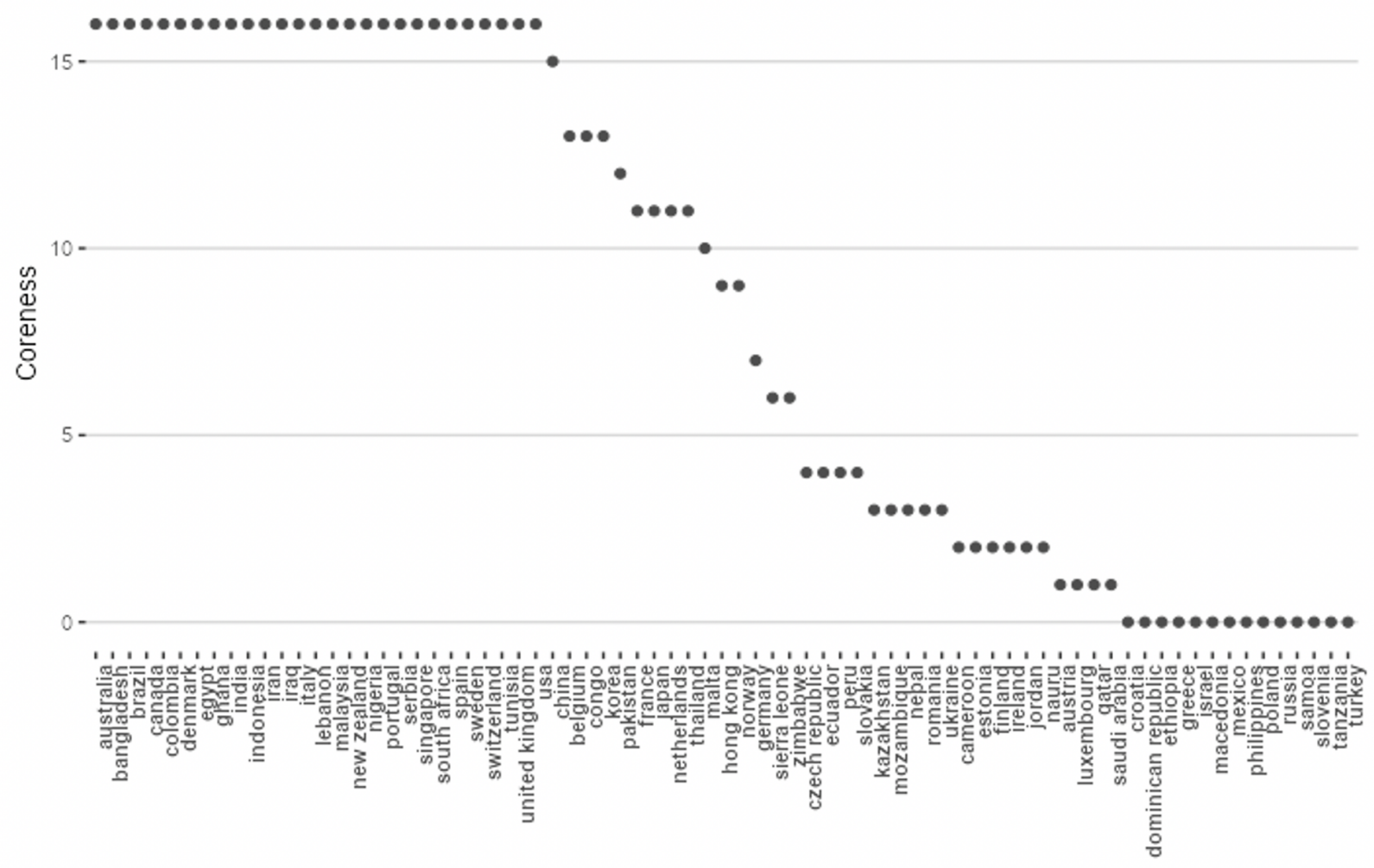

Figure 7: K-core structure of the country collaboration network.
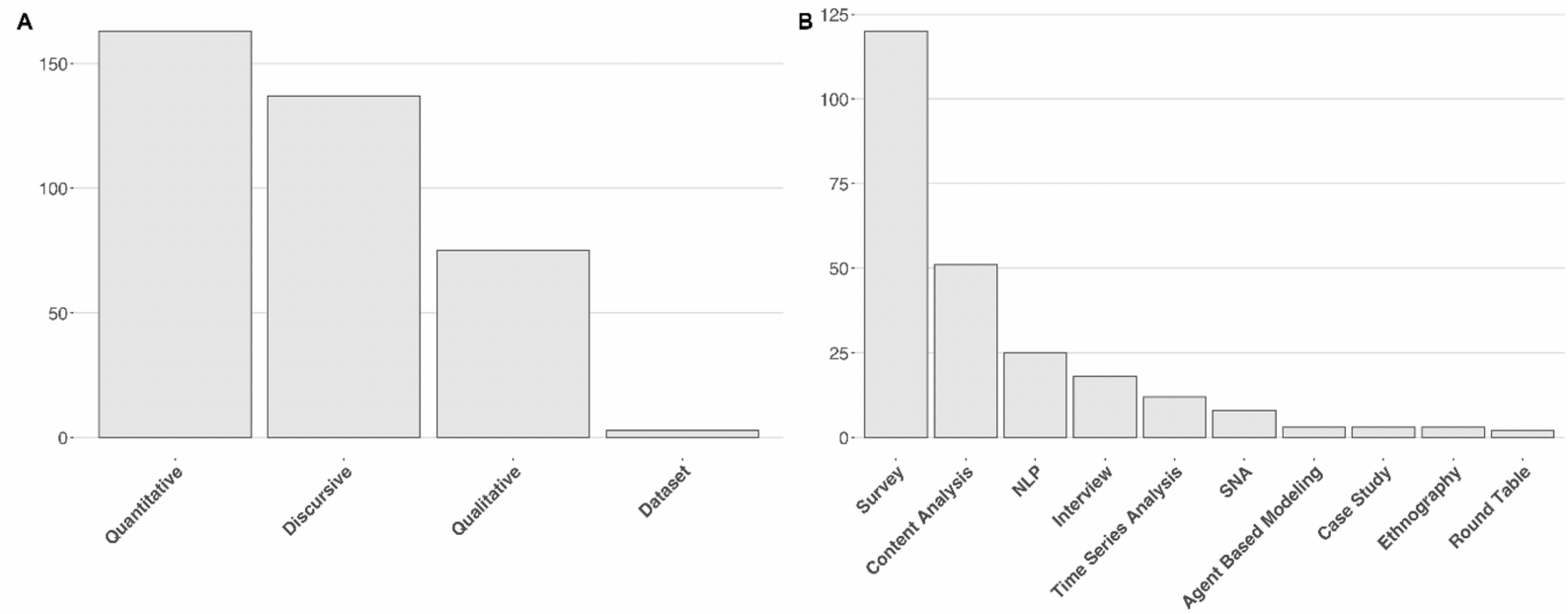

Figure 8: A) Frequency of approaches used in the papers on COVID-19 and problematic information (N=378). B) methods used in the empirical (quantitative and qualitative) papers $(\mathrm{n}=238)$. More than one method can be used in the same research. 


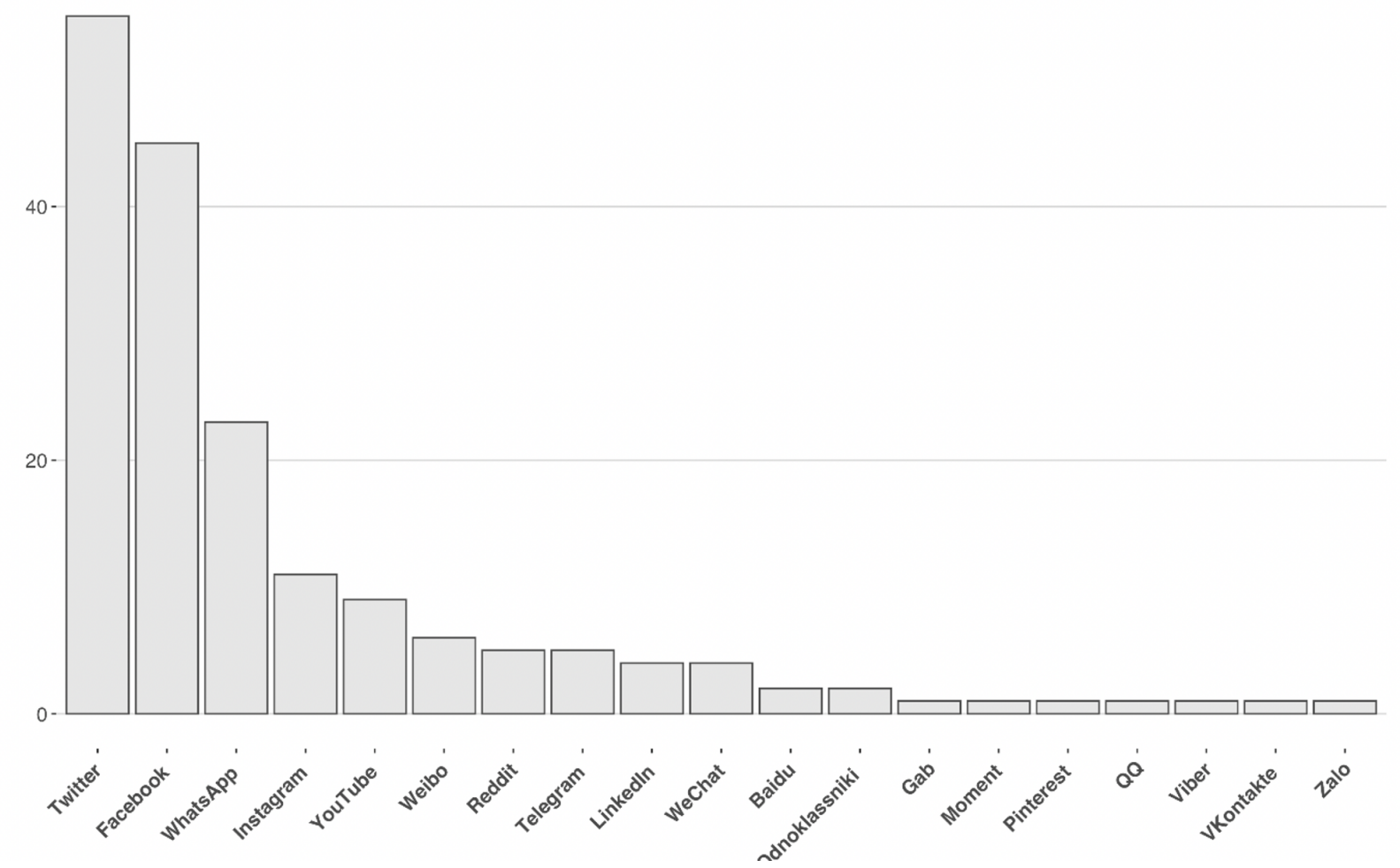

Figure 9: Frequency of social media platform occurrences (more than one platform can be used in the same study).

The sampling procedure is not the only important factor; the size of the sample is equally relevant to conducting proper statistical analyses. To calculate the sample size a method known as power analysis is commonly employed (Cohen, 1977). Survey-based research that made explicit reference to power analysis were $21.7 \%$. With regard to the size of the sample, considering the survey-based papers which we had access to and which specified the sample size, (113 out of 120,94\%), a total of 209,008 interviews were carried out.. Averaging the sample size used in the studies (certain studies ran more than one survey), they ranged from a minimum of 51 to a maximum of 40,157 interviewees, with a median value of 673.5 and a mean value of 1,503 $(\mathrm{sd}=3,953)$.

124 papers $(32.8 \%)$ included at least one social media platform in their research design. While this might appear surprising, considering that social media platforms have proven to be deeply intertwined with the spread of problematic information during critical social/political periods (Benkler, Faris, \& Roberts, 2018), such information is a multifaceted phenomenon and social media represents just one of its noteworthy aspects.

This list of social media platforms is dominated by Twitter, with a rating of almost $50 \%$, and Facebook, that is mentioned over 40 times. Likewise, mainstream social media platforms such as Instagram and YouTube received less attention, as well as instant messaging platforms (Figure 9).

\subsection{Substantive Content}

The analysis of the most frequently mentioned terms for problematic information in the title of the papers (176 papers, $47 \%$ of the corpus, mentioned at least one term in the title), revealed a few noticeable trends in literature (Fig. 10). First, a general preference emerges for widely used words like "misinformation" $(27 \%$ of papers mentioning a keyword in the title, and $15 \%$ of the papers in the corpus), and "fake news" (20\% and $11 \%$ respectively). This preference is understandable in the light of simple communicative intentions, namely to place papers within a clear research strand. Moreover, although misinformation means, strictly speaking, false information spread without intention, the word is also used in a more general fashion to talk about problematic and inaccurate information. This differentiates it from a word like "disinformation" which 


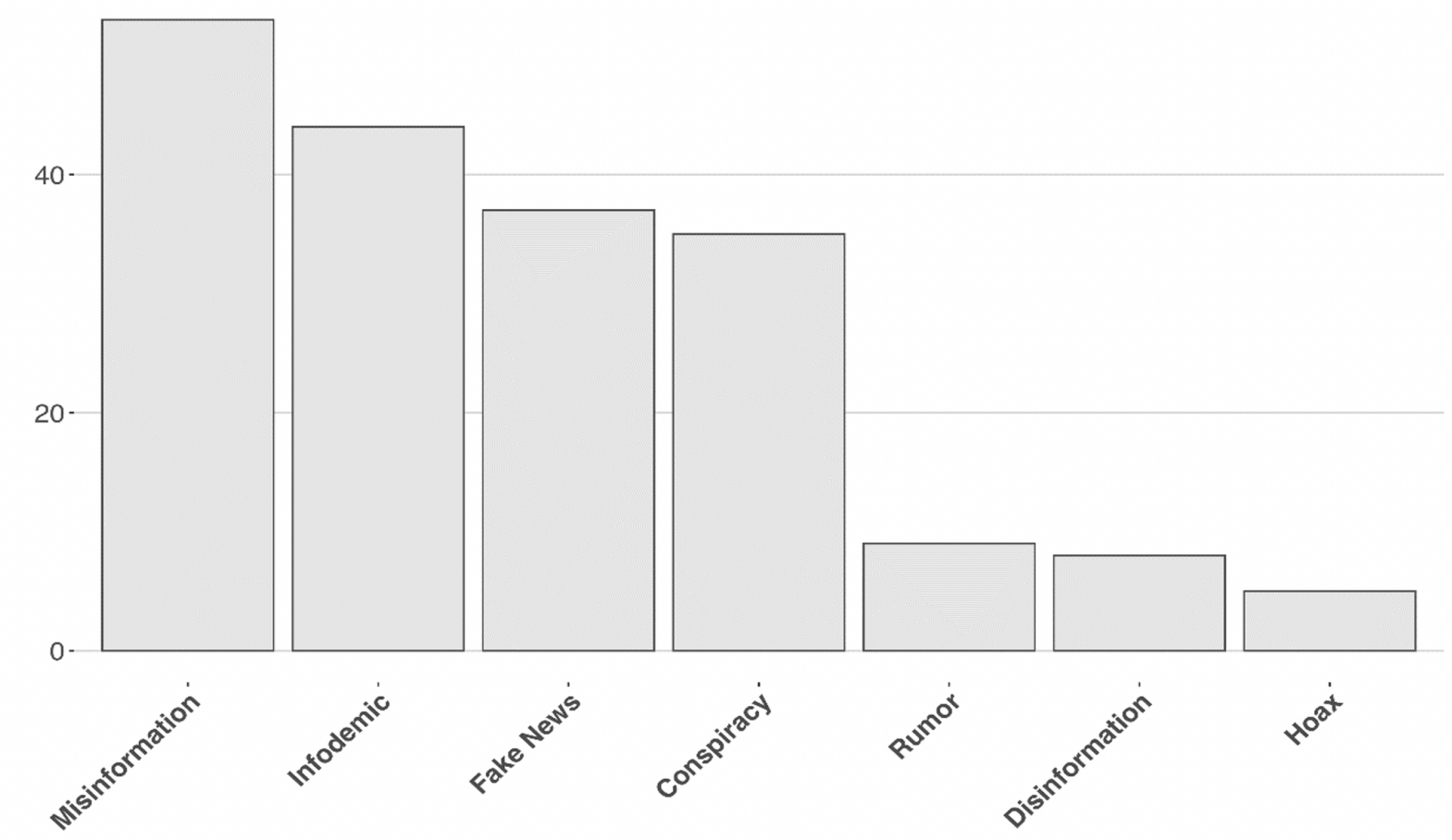

Figure 10: Terms for problematic information used in the title of the papers $(\mathrm{n}=176$ papers mentioning at least one keyword in the title).

appears far less often in the corpus and explicitly assumes the intentional spread of false information and potentially suggests the existence of information operations.

What is especially noticeable, however, is that the second most common keyword is "infodemic" (23\% of the papers using a keyword in the title, and $12 \%$ of the corpus). Ranking second among the most frequently used terms to refer to information-related problems in the context of the COVID-19 pandemic - even before widely used terms such as "misinformation" and "fake news" (Righetti, 2021) - the term confirms the considerable influence of The World Health Organization in setting the research agenda on the topic. By giving a name to the issue and drawing attention to it, infodemic has suddenly become an urgent problem to tackle. Although the term was coined before the COVID-19 pandemic (Rothkopf, 2003), its popularity in scientific research skyrocketed only in 2020: searching for every mention of the term in the full text of the documents included in the scientific database Scopus, there were no occurrences before 2019, just two entries in 2019 , and 792 in 2020 .

Conspiracy is the other relevant keyword that stands out in this first year of research on the pandemic $(18 \%$ of the keyword-in-title papers and $10 \%$ of the corpus), a relevance further confirmed by the results of the text analyses we are now going to describe.

The lexical analysis (Reinert, 1983, 1990) distinguished three main clusters of words. Each represents a "lexical world" and (they) together they summarize the semantics of the corpus (Figure 11). On the top-left quadrant are the terms depicting the more general context of the health emergency: "pandemic", "public", "health", "disease" "spread", "challenge", "society", "crisis", "global", "medium", "information", and "infodemic", are the eloquent words characterizing this cluster. The top-right quadrant encompasses the methodological dimension of the corpus: "survey", "analysis", "search", "participant", "questionnaire", "method", and "collect", are some representative words in this cluster. The third lexical cluster lies in the middle of the lower part of the Cartesian plan, focuses on the substantive content of the paper, and turns the spotlight on the centrality of conspiracy theories along with their consequences for compliance with health-protective measures such as social distancing and wearing masks. Among the representative words in this cluster are: "conspiracy theory", "belief", "perceive", "5G", "behavior", "distance", "motivation", "negatively", "positively", "predict", "compliance", and "wear". 


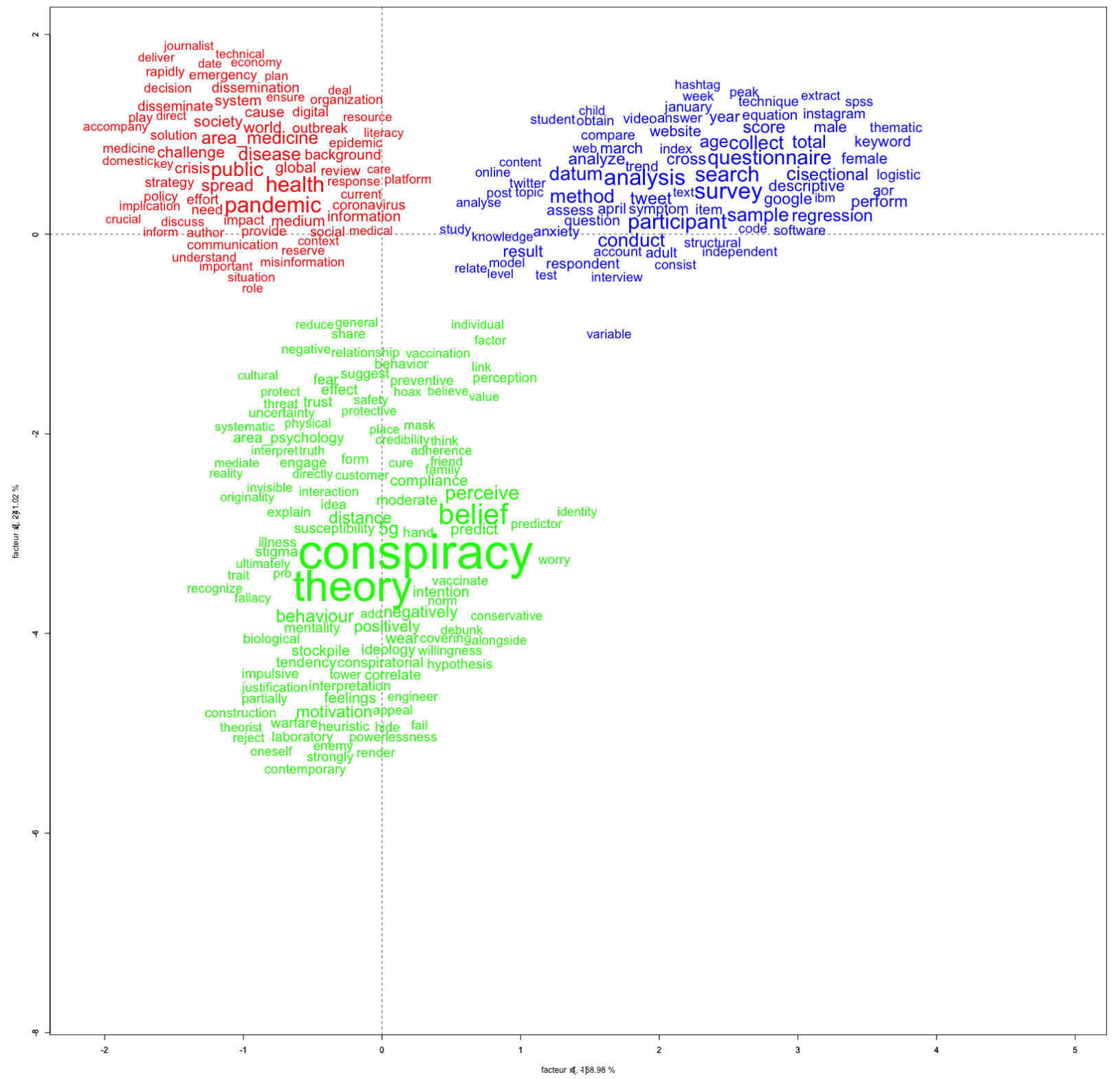

Figure 11: Reinert's Descending hierarchical classification. 
The attention to conspiracy theories and their impact on adherence to health-protective behaviors is further confirmed by the structural topic modeling analysis (Figure 12 and 13), which singled out this theme as the most prevalent in the corpus (Topic 3). Comparatively, other high frequency topics (e.g.: Topic 12 and 11) are marked by far more blurred boundaries, and their prevalence can therefore be considered as a side effect of their generality. On the contrary, the topic about conspiracy is both specific and widespread. The analysis of topic prevalence by disciplines (Figure 13) indicates the importance of psychological sciences which made a significant contribution to the understanding of conspiracy thinking well before the onset of the pandemic - in this area of research. Representative papers are: "Looking out for myself: Exploring the relationship between conspiracy mentality, perceived personal risk, and COVID-19 prevention measures", published in British Journal of Health Psychology (Marinthe et al., 2020), "Cultural orientation, power, belief in conspiracy theories, and intentions to reduce the spread of COVID-19", published in British Journal of Social Psychology (Biddlestone, Green, and Douglas, 2020), and "Association of COVID-19 misinformation with face mask wearing and social distancing in a nationally representative US sample", published in Health Communication (Hornik et al., 2021, online first in 2020), just to cite a few examples.

The consequences of misinformation have been considered in relation to behavior capable of limiting the contagion, like face masks and social distancing, but also as regards attitudes such as vaccine skepticism which obstruct measures potentially capable of stemming the pandemic (Topic 14). Account has been taken not only of health behaviors but also of behaviors impacting on the availability of goods on the market (Topic 10), investigating phenomena such as panic stockpiling (Naeem, 2020), and the fluctuating availability and prices of medicines. For instance, certain treatments like hydroxychloroquine raised controversies and misinformation flows, which led to shortages and price rises (Godman, 2020). Understandably, this topic was seized upon especially by economists (Figure 13).

Another strand of research was about drivers behind sharing false information (Topic 9). Representative papers are: "What drives unverified information sharing and cyberchondria during the COVID-19 pandemic?", published in European Journal of Information Systems (Laato et al., 2020), or "The spread of fake science: Lexical concreteness, proximity, misinformation sharing, and the moderating role of subjective knowledge", published in Public Understanding of Science (Kirkpatrick, 2020), to mention but a few.

A relevant strand of research focused on social media (e.g.: Topic 4, 6, 7, and 9), universally considered a major means of enabling an infodemic (Cinelli et al., 2020). Studies have described the main myths about the pandemic and analyzed the process of misinformation spreading (Gruzd \& Mai, 2020), differentiated false claims into separate categories (Islam et al., 2020), and tackled specific types of health misinformation for economic purposes, like that related to immune-boosting products (Rachul et al., 2020) and other questionable "cures" (Mackey et al. 2020). They have tried to assess the prevalence of misinformation (Li et al. 2020; Prabhakar \& Prasad, 2020), and to compare the popularity of scientific and fact-checked content (Pulido et al., 2020).

A relevant focus of interest has been the psychological and psychiatric consequences of the pandemic and misinformation, often analyzed in relation to social media (Topic 8). Although responsible for a considerable flow of problematic information online, research has pointed out the positive role of social media in pandemic communication (Lovari \& Righetti, 2021). For instance, it has been observed that celebrities can easily reach many followers with quality information (Mututwa, 2020), and while higher levels of social media use for both social support and information seeking are associated with higher levels of anxiety and depression (Drouin et al., 2020; Gao et al., 2020; Ni et al., 2020), social media can operate as allies of the institutions in reaching people with correct information, becoming protective agents supporting psychological well-being (Liu \& Tong, 2020).

The geographical areas representing the substantive focus of the research - either because the research analyzed that specific context or the subjects of the studies were from a specific region - partially reflect the structure of international collaboration highlighted in the analysis of the academic networks presented above, but also reveal differences. The higher level of international collaboration in the Medicine field is mirrored by the wider range of countries taken into consideration in the analyses (Figure 14), while Social Sciences have concentrated their efforts on a lower number of countries than Medicine, though still producing research spanning more countries than Psychology, the third most represented discipline in the corpus. From the substantive perspective considerered here, therefore, psychology showed the lowest degree of internationality. Using the Gini coefficient - the most commonly used measure of inequality, ranging from 0 to 1 (the higher the value, the more unequal the distribution) - as a measure of the geographical concentration of research interest, Medicine turns out to have, in fact, having a Gini equal to 0.376, Social Sciences equal to 0.430, and Psychology to 0.479. The results depend in part on the different levels of scientific productivity, since it is 


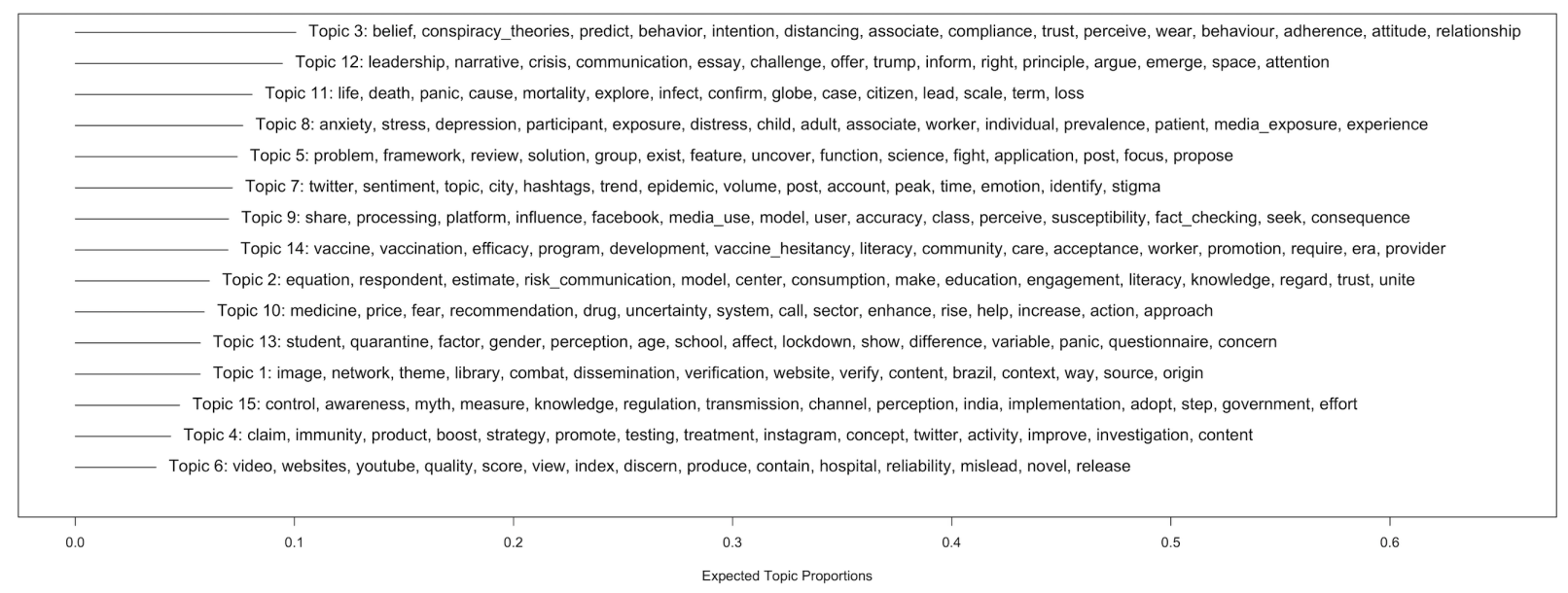

Figure 12: Most representative words by topic and expected topic proportions.
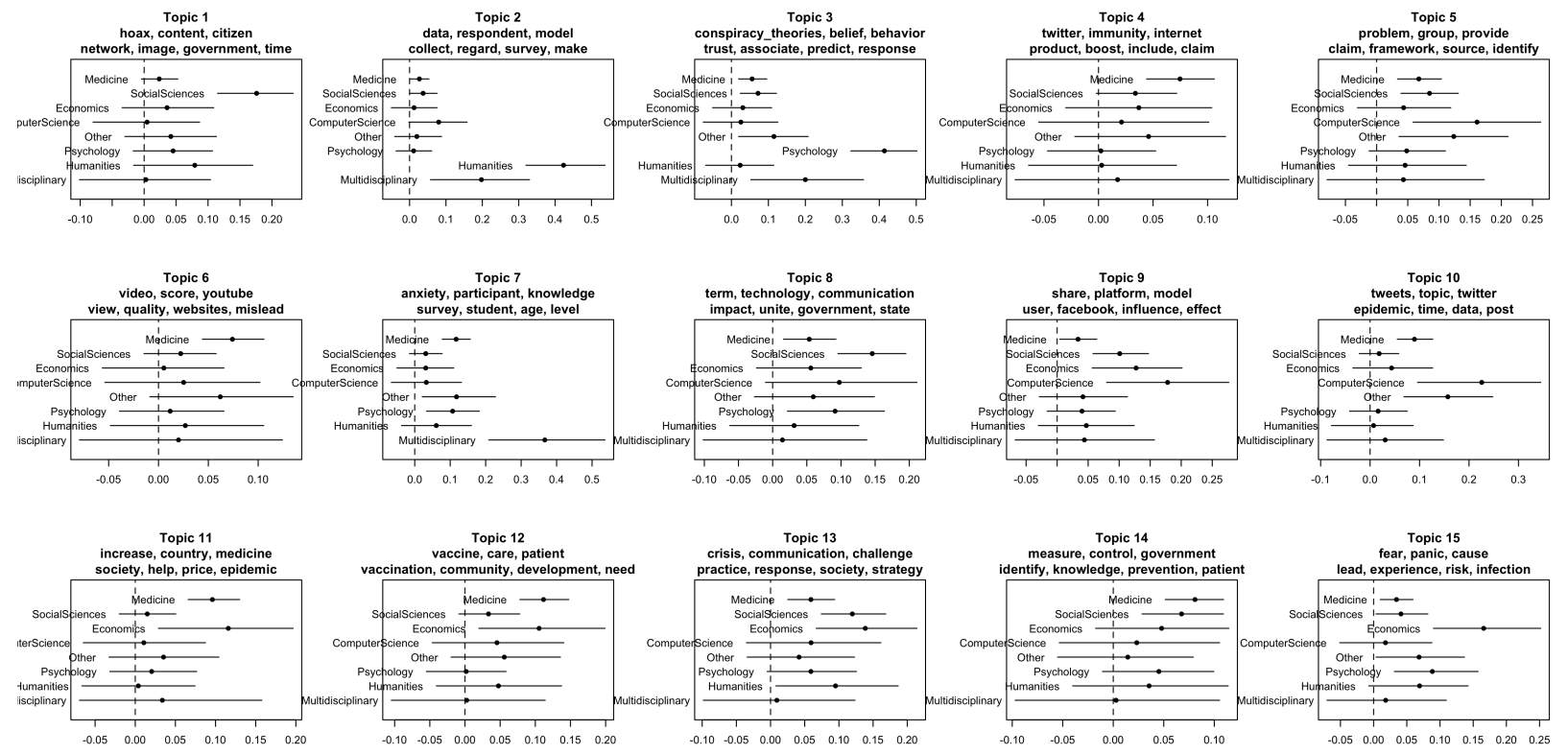

Figure 13: Topic proportions by research field. 

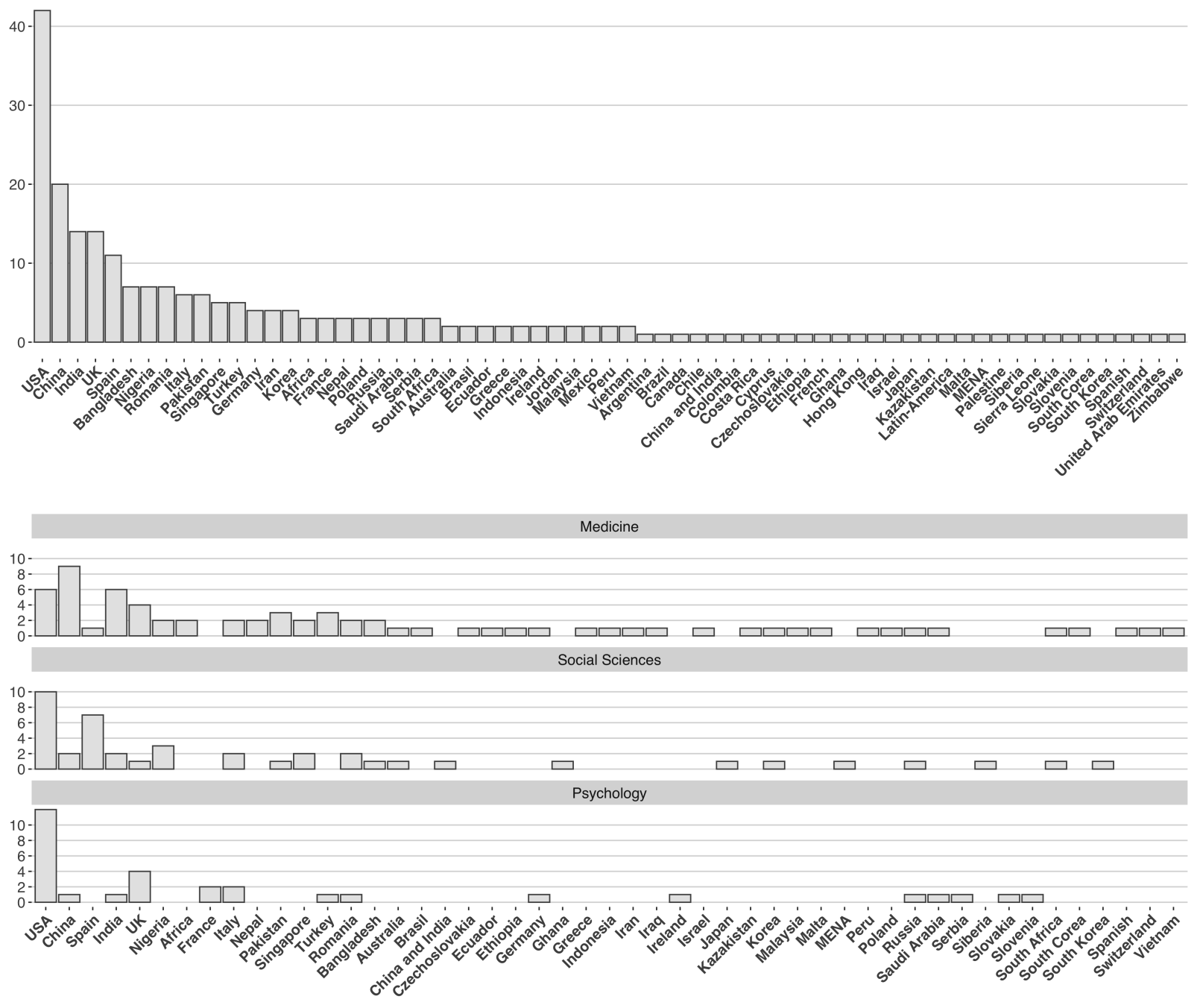

Figure 14: Countries mentioned in the papers.

impossible for a discipline producing just a few papers to analyze all the world's countries; but even within this limit, the unequal attention to certain geographical areas, such as USA, is clear.

\section{Discussion}

The literature review on problematic information and COVID-19, conducted following the PRISMA guidelines (Moher et al., 2009), resulted in a comprehensive corpus of 378 papers on the topic, published in 2020. We analyzed this large corpus of research from a tridimensional perspective, taking into account the disciplinary structure of the field and the collaboration among scholars from different parts of the world, the methods used in the research, and the main trends in the substantive content of the research. We proceeded to analyze the data by using a two-step process. We first conducted a qualitative analysis of the corpus during which we coded the papers following a detailed schema, to allow relevant information to emerge concerning the aforementioned dimensions. Then, we adopted a quantitative, computational approach including social network analysis and text mining to analyze the data.

Misinformation is a worldwide phenomenon and information-related problems are multifaceted, thus a multidisciplinary and cross-national approach is necessary to analyze them in all their complexity and nuances. We conducted a thorough analysis of the disciplinary networks and the relations between disciplines using a network analysis approach, focusing first on the analysis of co-citations, and then on scholars' international 
collaborations. We found that most of the articles were published within disciplinary journals but that a large number of disciplines have worked on the problem, with Medicine and Social Sciences taking the lion's share. Moreover, looking at the literature cited by the papers in our dataset we can say that disciplinary confines do not strictly bound the scientific horizon of scholars, but papers are linked together beyond disciplinary boundaries by a common set of references. We also found different degrees of international collaboration between scholars, from disciplines that rely strongly on international networks, such as Medicine, to less internationally structured disciplines, such as Social Sciences. It also emerged that, besides stronger links such as those between USA and UK, regional factors do not impact the actual structure of international networks, meaning that, with the exception of a few cases, , international networks are not structured on the basis of a strong and discernible geographical dimension. This networked geographical analysis was complemented by the analysis of the countries mentioned in the papers - either because of the geographical context of research or because of the country the studied subjects (e.g.: surveys' interviewees) come from finding a skewed distribution of interest across countries. There is a stronger focus on the USA, but many papers also dealt with China, where the virus first originated. The USA-centrism characterizes the entire corpus and is particularly evident in Psychology, while other important disciplines in the dataset, like Social Sciences and especially Medicine, took a larger range of countries into account in their analyses.

Considering the methodological approaches, the higher share of quantitative methodologies can be interpreted in the light of their traditionally representing one of the most widespread approaches in use in the social sciences as well as in health-related fields (e.g.: epidemiology) ; but the restrictions on movement, in the wake of the pandemic, also made methods like self-administered surveys - which represent the lion's share of research in the corpus ( $49 \%$ of the empirical papers) - easier than those based on qualitative approaches such as interviews or ethnographic observation. Qualitative approaches, moreover, tend to be more expensive in terms of time, and therefore more difficult to adopt when the available time is scarce, like during an emergency. Discursive papers, which are the second most frequent approach, provide informed analysis, sometimes proposing strategies, recommendations, and possible solutions to help tackle field-specific problems created by the pandemic. Considering the urgent need for expert views in the extraordinary circumstances brought about by the pandemic, it is understandable that this is a major category in the corpus.

Certain methodological observations can also be made. For instance, regarding the many surveys that exploited social media or online services such as Amazon Mechanical Turk for collecting data, it can be observed that the approach successfully meets the need to collect evidence as soon as possible in the middle of the pandemic, but the convenient, self-selected samples collected in this way are usually unrepresentative and potentially biased. Moreover, just $21.7 \%$ of the papers based on surveys make reference to power analysis, necessary to ensure statistically significant results, although the sample size used in the research is usually not too small $(1,503$ on average, with a median of 673.5$)$. Nonetheless, the data collected through this approach can be relevant, and a systematic meta-analysis could be used to reach a synthesis and more robust results. A similar meta-analysis would be useful with regard to results from social media research. The textual analysis, which was conducted with a variety of approaches to ensure more robust results, clearly highlighted conspiracy theories and their impact on compliance with health-protective behaviors as the most significant trend in the first year of study of problematic information around COVID-19. Research has also considered the problem from the perspective of its consequences on other spheres of social and individual life, such as psychological well-being or market dynamics. Besides consequences, other topics such as motives for sharing false or misleading information, the process of misinformation spreading online, the overall prevalence of the problem and types of misleading content, and a wide range of possible solutions have also been proposed.

A second noticeable trend of the 2020 research on pandemic-related information problems was the so-called infodemic. The term is the second most popular keyword in the titles of papers on the topic. Despite being coined several years ago, it skyrocketed in academic research only in 2020, after the WHO defined COVID-19 as a pandemic and an infodemic. The popularity of the term suggests the power of the World Health Organization in setting the research agenda, and the considerable amount of COVID-19-related literature on infodemic could prove instrumental in establishing a new research tradition in the field of problematic information and health sciences, namely information epidemiology or infodemiology, "The science of distribution and determinants of information in an electronic medium, specifically the Internet, or in a population, with the ultimate aim to inform public health and public policy" (Eysenbach, 2009; Mavragani, 2020). Generally speaking, terms employed to define a situation carry assumptions and frames with them, sometimes emphasizing particular paths to solutions. In the case of infodemic, the term underlines a medical paradigm, implicitly defining information problems in pathological terms. Scholars might find this approach more or less stimulating and useful, but a thorough analysis of the term's uses and related theoretical assumptions could be a useful way to take a step back and critically reflect on the direction given to the field. 


\section{Conclusions}

We conducted a systematic comprehensive literature review of research on problematic information around COVID-19 published in 2020, the first year of the pandemic. A tridimensional analysis on 378 papers was conducted to investigate the scientific structure of the field, the methodological choices, and the substantive content, identifying the main trends. We categorized the papers and used frequencies and computational methods like social network analysis and text mining to analyze the data.

Findings revealed a disciplinarily interconnected and geographically heterogeneous field of study with considerable contributions from Medicine and Social Sciences. Quantitative methods and especially surveys were among the mostly commonly used approaches, with a substantial number of discursive papers offering expert views on pandemic-related informational problems. The main trends, from a substantive perspective, were conspiracy theories and their impact on norm compliance, and the attention to informational problems defined through the concept of infodemic. The literature review served to bring a number of considerations into sharp focus: it underlined the value of interdisciplinarity to tackle the multifaceted issue of problematic information, the importance of paying due attention to a variety of geographical and cultural contexts and social media platforms, the relevance of careful attention to the quality of sampling and statistical analysis, the usefulness of further meta-analysis to synthesize, integrate and increase the robustness of already established results in the literature, and the potential significance of a critical scrutiny of terminologies and related assumptions used in structuring the field.

\section{References}

Allcott, H., \& Gentzkow, M. (2017). Social media and fake news in the 2016 election. Journal of economic perspectives, $31(2), 211-36$.

Allington, D., \& Dhavan, N. (2020). The relationship between conspiracy beliefs and compliance with public health guidance with regard to COVID-19. https://kclpure kcl. ac. uk/portal/files/127048253/Allington_and_Dhavan_2020. pdf.

Benkler, Y., Faris, R., \& Roberts, H. (2018). Network propaganda: Manipulation, disinformation, and radicalization in American politics. Oxford University Press.

Bermejo, F. (2020). Information Pandemic: Initial explorations of COVID19 coverage. Media Cloud. https://mediacloud.org/news/2020/3/22/ information-pandemic-initial-explorations-of-covid-19-coverage

Biddlestone, M., Green, R., \& Douglas, K. M. (2020). Cultural orientation, power, belief in conspiracy theories, and intentions to reduce the spread of COVID-19. British Journal of Social Psychology, 59(3), 663-673.

Brennen, J. S., Simon, F. M., Howard, P. N., \& Nielsen, R. K. (2020). Types, Sources, and Claims of COVID-19 Misinformation. Reuters Institute, 13. https://reutersinstitute.politics.ox.ac.uk/ types-sources-and-claims-covid-19-misinformation

Chan, C. H., \& Grill, C. (2020). The highs in communication research: Research topics with high supply, high popularity, and high prestige in high-impact journals. Communication Research, 0093650220944790.

Cinelli, M., Quattrociocchi, W., Galeazzi, A., Valensise, C. M., Brugnoli, E., Schmidt, A. L., ... \& Scala, A. (2020). The covid-19 social media infodemic. Scientific Reports, 10(1), 1-10.

Cohen, J. (1977). Statistical power analysis for the behavioral sciences. Academic press. New York, San Francisco, London.

DiResta, R., Milller, C., Molter, V., Pomfret, J., \& Tiffert, G. (2020). Telling China's Story: The Chinese Communist Party's Campaign to Shape Global Narratives. Stanford Internet Observatory. https://cyber. fsi.stanford.edu/io/news/new-whitepaper-telling-chinas-story

Douglas, K. M. et al. 2019. "Understanding Conspiracy Theories." Political Psychology, 40: 3-35.

Drouin, M., McDaniel, B. T., Pater, J., \& Toscos, T. (2020). How parents and their children used social media and technology at the beginning of the COVID-19 pandemic and associations with anxiety. Cyberpsychology, Behavior, and Social Networking, 23(11), 727-736. 
Eysenbach, G. (2009). Infodemiology and infoveillance: framework for an emerging set of public health informatics methods to analyze search, communication and publication behavior on the Internet. Journal of medical Internet research, 11(1), e11.

Ferrara, E., Cresci, S., \& Luceri, L. (2020). Misinformation, manipulation, and abuse on social media in the era of COVID-19. Journal of Computational Social Science, 1-7.

Gao, J., Zheng, P., Jia, Y., Chen, H., Mao, Y., Chen, S., .. \& Dai, J. (2020). Mental health problems and social media exposure during COVID-19 outbreak. Plos one, 15(4), e0231924.

Giglietto, F., Righetti, N., \& Marino, G. (2020). Detecting Coordinated Link Sharing Behavior on Facebook during the Italian Coronavirus Outbreak. AoIR Selected Papers of Internet Research. https://www.spir. aoir.org/ojs/index.php/spir/article/view/11219

Giglietto, F., Marino, G., Terenzi, M., Righetti, N., \& Rossi, L. (2021). Coordinated Hateful Disinformation on Italian Politics and Social Issues, since 2017. Available at SSRN 3777263. https://papers.ssrn.com/ sol3/papers.cfm?abstract_id $=3777263$

Godman, B., Haque, M., Islam, S., Iqbal, S., Urmi, U. L., Kamal, Z. M., . . \& Hossain, M. M. (2020). Rapid assessment of price instability and paucity of medicines and protection for COVID-19 across Asia: findings and public health implications for the future. Frontiers in public health, 8 .

Grey Ellis, E. 2020. The Coronavirus Outbreak Is a Petri Dish for Conspiracy Theories. Wired.

Gruzd, A., \& Mai, P. (2020). Going viral: How a single tweet spawned a COVID-19 conspiracy theory on Twitter. Big Data \& Society, 7(2), 2053951720938405.

Hornik, R., Kikut, A., Jesch, E., Woko, C., Siegel, L., \& Kim, K. (2021). Association of COVID-19 misinformation with face mask wearing and social distancing in a nationally representative US sample. Health communication, 36(1), 6-14

Islam, M. S., Sarkar, T., Khan, S. H., Kamal, A. H. M., Hasan, S. M., Kabir, A., .. \& \& Seale, H. (2020). COVID-19-related infodemic and its impact on public health: A global social media analysis. The American Journal of Tropical Medicine and Hygiene, 103(4), 1621.

Jack, C. (2017). Lexicon of lies: Terms for problematic information. Data \& Society, 3, 22. Chicago

Kessler, M. M. (1963). Bibliographic coupling between scientific papers. American documentation, 14(1), $10-25$.

Kirkpatrick, W. A. (2020). The spread of fake science: Lexical concreteness, proximity, misinformation sharing, and the moderating role of subjective knowledge. Public Understanding of Science, 0963662520966165.

Krippendorff, K. (2004). Content analysis: An introduction to its methodology. Thousand Oaks, California: Sage.

Laato, S., Islam, A. N., Islam, M. N., \& Whelan, E. (2020). What drives unverified information sharing and cyberchondria during the COVID-19 pandemic? European Journal of Information Systems, 29(3), 288-305

Lasco, G. (2020). Medical populism and the COVID-19 pandemic. Global Public Health, 15(10), 1417-1429.

Li, H. O. Y., Bailey, A., Huynh, D., \& Chan, J. (2020). YouTube as a source of information on COVID-19: a pandemic of misinformation? BMJ global health, 5(5).

Lovari, A. (2020). Spreading (dis) trust: Covid-19 misinformation and government intervention in Italy. Media and Communication, 8(2), 458-461.

Lovari, A., Martino, V., \& Righetti, N. (2019). Blurred Shots: Investigating the Information Crisis Around Vaccination in Italy. American Behavioral Scientist, 0002764220910245.

Lovari, A., Righetti, N., (2021), "Fake News and Pandemic Communication", in Crawford P., Kadetz, P.I. (eds), Palgrave Encyclopedia of the Health Humanities.

Mackey, T. K., Li, J., Purushothaman, V., Nali, M., Shah, N., Bardier, C., . . \& Liang, B. (2020). Big Data, Natural Language Processing, and Deep Learning to Detect and Characterize Illicit COVID-19 Product Sales: Infoveillance Study on Twitter and Instagram. JMIR public health and surveillance, 6(3), e20794 
Marinthe, G., Brown, G., Delouvée, S., \& Jolley, D. (2020). Looking out for myself: Exploring the relationship between conspiracy mentality, perceived personal risk, and COVID-19 prevention measures. British Journal of Health Psychology, 25(4), 957-980.

Mavragani, A. (2020). Infodemiology and infoveillance: scoping review. Journal of medical Internet research, 22(4), e16206.

Molter, V., \& DiResta, R. (2020). Pandemics \& propaganda: How Chinese state media creates and propagates CCP coronavirus narratives. Harvard Kennedy School Misinformation Review, 1(3).

Mututwa, W. (2020). COVID-19 infections on international celebrities: self-presentation and tweeting down pandemic awareness. Journal of Science Communication, 19(5).

Naeem, M. (2020). The role of social media to generate social proof as engaged society for stockpiling behaviour of customers during Covid-19 pandemic. Qualitative Market Research: An International Journal.

Neumayer, C., \& Rossi, L. (2016). 15 years of protest and media technologies scholarship: a sociotechnical timeline. Social Media+Society, 2(3), 2056305116662180.

Newman, M. E. (2002). Assortative mixing in networks. Physical review letters, 89(20), 208701.

Ni, M. Y., Yang, L., Leung, C. M., Li, N., Yao, X. I., Wang, Y., .. \& Liao, Q. (2020). Mental health, risk factors, and social media use during the COVID-19 epidemic and cordon sanitaire among the community and health professionals in Wuhan, China: cross-sectional survey. JMIR mental health, 7(5), e19009.

Page, M. J., Moher, D., Bossuyt, P. M., Boutron, I., Hoffmann, T. C., Mulrow, C. D., .. \& \& McKenzie, J. E. (2021). PRISMA 2020 explanation and elaboration: updated guidance and exemplars for reporting systematic reviews. BMJ, 372.

Prabhakar Kaila, D., \& Prasad, D. A. (2020). Informational flow on Twitter-Corona virus outbreak-topic modelling approach. International Journal of Advanced Research in Engineering and Technology (IJARET), 11(3).

Pulido, C. M., Villarejo-Carballido, B., Redondo-Sama, G., \& Gómez, A. (2020). COVID-19 infodemic: More retweets for science-based information on coronavirus than for false information. International Sociology, 35(4), 377-392.

Rachul, C., Marcon, A. R., Collins, B., \& Caulfield, T. (2020). COVID-19 and 'immune boosting' on the internet: a content analysis of Google search results. BMJ, 10(10), e040989.

Ratinaud, P. (2008). IRAMUTEQ (Interface de R pour les Analyses Multidimensionnelles de Textes et de Questionnaires). http://www.iramuteq.org

Reinert M. (1983). Une méthode de classification descendante hiérarchique : application à l'analyse lexicale par contexte. Cahiers de l'analyse des données, Volume 8, Numéro 2. http://www. numdam.org/item/?id= CAD_1983_-8_2_187_0

Reinert M. (1990). Alceste une méthodologie d'analyse des données textuelles et une application: Aurelia De Gerard De Nerval. Bulletin de Méthodologie Sociologique, Volume 26, Numéro 1. https://doi.org/10. $1177 / 075910639002600103$

Righetti, N. (2021). The Impact of the Politicization of Health on Online Misinformation and Quality Information on Vaccines. Italian Sociological Review, 11 (2), 443-466.

Righetti, N. (2021). Four Years of Fake News. A Quantitative Analysis of the Scientific Literature. First Monday, 26(6-7), June 2021.

Roberts, M. E., Stewart, B. M., \& Tingley, D. (2019). Stm: An R package for structural topic models. Journal of Statistical Software, 91(1), 1-40.

Serrato, R., \& Schafer, B. (2020). Reply All: Inauthenticity and Coordinated Replying in Pro-Chinese Communist Party Twitter Networks. ISD.

Shahsavari, S., Holur, P., Tangherlini, T. R., \& Roychowdhury, V. (2020). Conspiracy in the Time of Corona: Automatic detection of Covid-19 Conspiracy Theories in Social Media and the News. ArXiv:2004.13783.

Stanyer, J. (2009). Web 2.0 and the transformation of news and journalism. Routledge Handbook of Internet Politics, 201 
Subramanian, S. (2017). Inside the Macedonian fake-news complex. Wired, 15.

Sunstein, C. R. (2018). \#Republic: Divided democracy in the age of social media. Princeton University Press.

Sunstein, C.R., Vermeule, A. (2009). Conspiracy Theories: Causes and Cures. Journal of Political Philosophy, 17(2): 202-27.

Swami, V., David B. (2020). Analytic Thinking, Rejection of Coronavirus (COVID-19) Conspiracy Theories, and Compliance with Mandated Social-Distancing https://osf .io/nmx9w/ (May 7, 2020).

Wang, Y., McKee, M., Torbica, A., \& Stuckler, D. (2019). Systematic literature review on the spread of health-related misinformation on social media. Social Science \& Medicine, 240, 112552.

Webster, J., \& Watson, R. T. (2002). Analyzing the past to prepare for the future: Writing a literature review. MIS quarterly, xiii-xxiii.

WHO (2020). Director-General's opening remarks at the media briefing on COVID-19 - 11 March 2020. https://www.who.int/dg/speeches/detail/ who-director-general-s-opening-remarks-at-the-media-briefing-on-covid-19---11-march-2020

Zarocostas, J. (2020). How to fight an infodemic. Lancet, 395(10225), 676. https://doi.org/10.1016/ S0140-6736(20)30461-X 